Revista Destaques Acadêmicos, Lajeado, v. 9, n. 4, 2017. ISSN 2176-3070 DOI: http://dx.doi.org/10.22410/issn.2176-3070.v9i4a2017.1666 www.univates.br/revistas

\title{
INTEGRAÇÃO DE DIFERENTES FERRAMENTAS PARA O ESTUDO DO COMPORTAMENTO ELETROMAGNÉTICO EM MATERIAIS MACIOS SINTERIZADOS APLICADOS EM MÁQUINAS ELÉTRICAS
}

\author{
Mateus Vorpagel ${ }^{1}$, Ederson Pereira Madruga ${ }^{2}$, Jaime Back ${ }^{3}$, Lírio Schaeffer ${ }^{4}$
}

\begin{abstract}
Resumo: O presente trabalho tem como objetivo apresentar a integração de diferentes ferramentas para o estudo do comportamento eletromagnético em materiais macios sinterizados, que são aplicados em máquinas elétricas, como motores e transformadores. Através da utilização do software LabVIEW, desenvolveu-se um aplicativo de instrumentação virtual capaz de analisar as curvas de histerese de diferentes materiais eletromagnéticos, como núcleos de transformadores sinterizados. Para desenvolvimento deste projeto utilizou-se uma placa modelo PCIe-6341 em conjunto com um módulo de entradas e saídas SCB-68, do fabricante da National Instruments. Para complementar, realizaram-se simulações destes materiais nos softwares FEMM 4.2 e PSIM 9.0, para determinação dos campos magnéticos e levantamento das curvas de histerese e posterior validação da proposta. Como resultados, foram comparadas as curvas de histerese e os valores de campo magnético e densidade de fluxo magnético do material testado pelo aplicativo desenvolvido no LabVIEW, com um traçador de curvas de histerese comercial modelo TLMP-FLX-D fabricado pela GLOBALMAG.
\end{abstract}

Palavras-chave: Máquinas elétricas. Materiais magnéticos. Curva de histerese. Instrumentação virtual.

1 Acadêmico do curso de Engenharia Elétrica da Universidade do Vale do Taquari - Univates. vorpa@universo.univates.br

2 Doutor e professor do curso de Engenharia Elétrica da Universidade Vale do Taquari Univates. ederson.madruga@univates.br

3 Doutorando no Depto. de Metalurgia (PPGE3M) da Universidade Federal do Rio Grande do Sul - UFRGS. jaime.back@gmail.com

4 Doutor e professor no Depto. de Metalurgia (PPGE3M) Universidade Federal do Rio Grande do Sul - UFRGS. schaefer@ufrgs.br 


\section{INTRODUÇÃO}

Buscando novos materiais e aplicações, pesquisadores vêm desenvolvendo e produzindo materiais magnéticos de baixo custo, que atendam as características mínimas necessárias para serem utilizados em máquinas elétricas. Para conseguir resultados satisfatórios, vem sendo testadas diferentes ligas metálicas, a fim de substituir os materiais mais comuns utilizados em máquinas elétricas (ROCHI, 2015).

Dentre os materiais estudados estão os materiais amorfos, materiais compósitos magnéticos macios e materiais nanocristalinos. O processo que se busca estudar é o Processo de Fabricação por Metalurgia do Pó (M/P). Este processo busca desenvolver novas ligas de materiais que apresentem maior rendimento, eficiência e menor custo de fabricação de máquinas elétricas. $\mathrm{O}$ processo de $\mathrm{M} / \mathrm{P}$ envolve as etapas de preparação da liga, moagem, compactação e sinterização. Seu custo é inferior, quando comparado com outros processos de fabricação, como por exemplo, chapas laminadas (LUNA, 2012; CERVA, 2014; ROCHI, 2015).

Segundo Dias (2005), estudos apontam que os materiais magnéticos sinterizados podem ser aproveitados para construir núcleos de máquinas elétricas, como motores e transformadores. Desta forma, as ligas de materiais magnéticos macios, que são constituídas de misturas de pós de ferro com fósforo, silício ou níquel seriam aplicadas em máquinas elétricas de núcleos maciços, substituindo as chapas de aço laminadas.

Com o intuito de contribuir para esta área de estudos, desenvolveu-se um aplicativo de instrumentação virtual no software LabVIEW, capaz de medir curvas de histerese. Esta proposta de trabalho surgiu como alternativa aos equipamentos comerciais existentes no mercado, geralmente de custo elevado. O LabVIEW permite que o usuário desenvolva aplicações personalizadas para um determinado fim, utilizando uma placa de aquisição de dados PCIe-4361 e uma interface de programação gráfica modular. A Figura 1 apresenta esta plataforma de instrumentação virtual. 
Figura 1 - Plataforma de instrumentação virtual

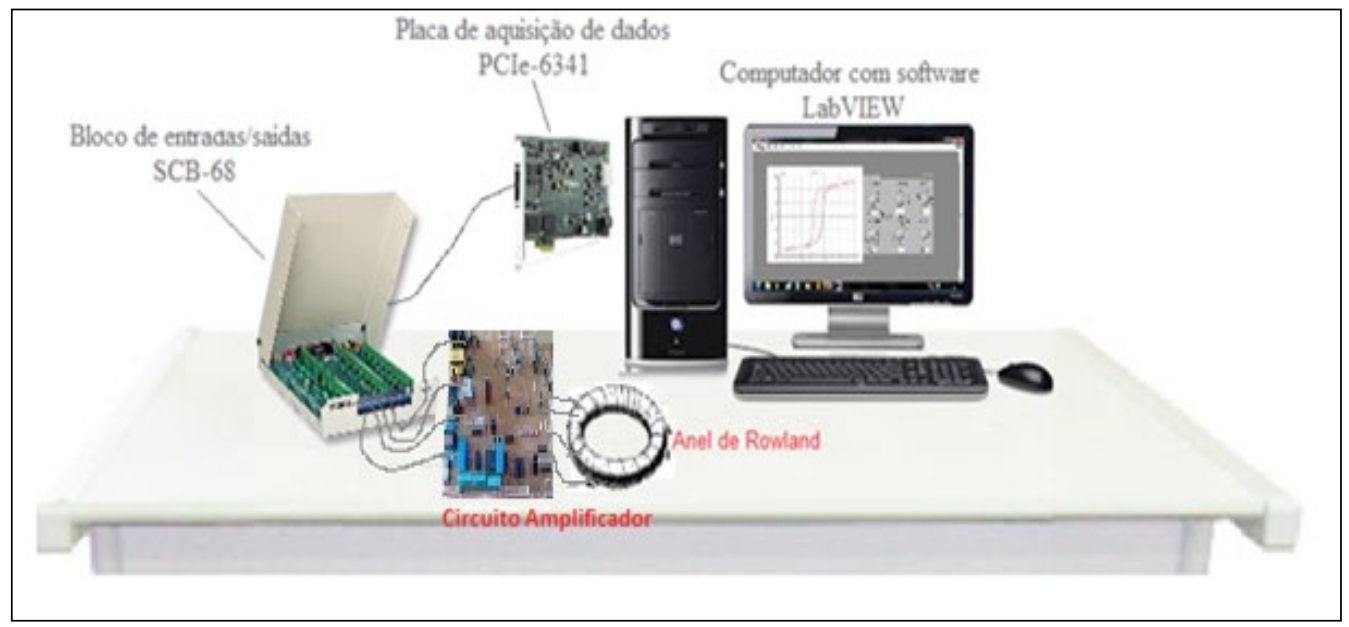

Fonte: Adaptado de BARBOSA (2016).

Para validação da proposta, comparou-se as curvas de histerese e os valores de campo magnético e densidade de fluxo magnético dos materiais testados pelo aplicativo desenvolvido no LabVIEW, com o Traçador de Curvas de Histerese da GLOBALMAG, modelo TLMP-FLX-D, e os resultados encontrados nos softwares FEMM 4.2 e PSIM 9.0. De acordo com Bianchini (2006), utilizar softwares simuladores, como FEMM e PSIM, é considerado uma alternativa na falta de equipamentos, geralmente de valor e custo elevado, como um histeresímetro ou um magnetizador.

Este artigo está organizado da seguinte forma: o segundo item apresenta a revisão bibliográfica sobre eletromagnetismo, curva de histerese, materiais moles e duros, materiais magnéticos compósitos, LabVIEW, FEMM e PSIM. O item três apresenta os materiais e métodos utilizados no desenvolvimento da plataforma de instrumentação virtual, como funcionamento do hardware utilizado para ler os sinais aplicados nos materiais e o funcionamento do software de instrumentação virtual. $\mathrm{O}$ item quatro 4 apresenta os resultados e as conclusões obtidas neste trabalho, como as curvas de histerese e o comportamento do material.

\section{REVISÃO}

\subsection{Eletromagnetismo}

De acordo com Griffiths (2012), todos os fenômenos magnéticos são devidos às cargas elétricas que estão em movimento. Analisando uma porção microscópica de material magnético, se encontraria elétrons orbitando em volta dos núcleos e elétrons girando pelo seu próprio eixo. Quando um campo 
magnético é aplicado nestes elétrons, ocorre o alinhamento líquido dos dipolos magnéticos e o meio se torna magnetizado. A polarização elétrica se dá na direção do campo elétrico. Materiais que possuem magnetização paralela ao campo magnético $B$ são chamados de paramagnéticos. Já materiais que possuem magnetização contrária ao campo magnético $B$ são chamados de ferromagnéticos. Callister (2007) compara os dipolos magnéticos como imãs de barras compostos de polo norte e sul, ao contrário de carga elétrica positiva e negativa. Esses dipolos são influenciados por campos magnéticos e a força desses campos exerce um torque capaz de orientá-los ao redor do campo magnético. A Figura 2 apresenta a distribuição do campo magnético de uma bobina e um imã indicada por linhas de força.

Figura 2 - Distribuição do campo magnético de uma bobina e um imã indicada por linhas de força

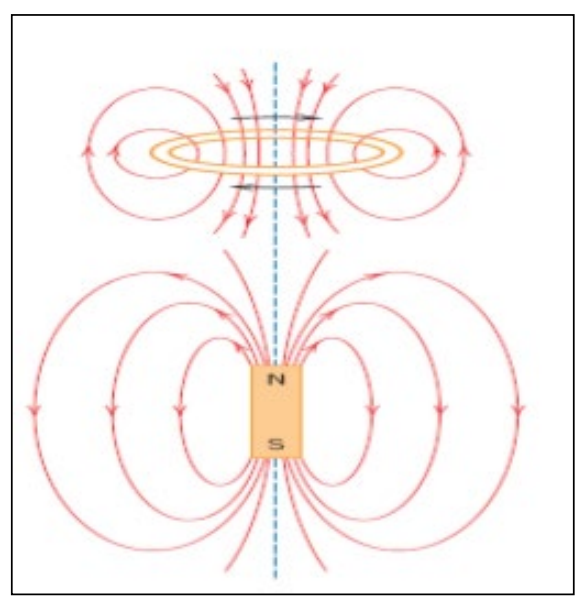

Fonte: CALLISTER (2007).

\subsubsection{Curva de histerese e ponto de Curie}

Temperaturas altas destroem o alinhamento paralelo dos dipolos dentro de um determinado domínio. No ferro essa temperatura é de $770^{\circ} \mathrm{C}$. Abaixo dessa temperatura que é chamada de ponto de Curie, o ferro é ferromagnético, acima, ele é paramagnético. $\mathrm{O}$ ponto de Curie não possui transição gradual entre o comportamento ferromagnético e paramagnético. Tais mudanças são conhecidas como transição de fases (GRIFFITHS, 2012).

Segundo Nasar (1987), a temperatura de Curie é a temperatura na qual os imãs se desmagnetizam. Para Cullity (1967), ao colocar um material ferromagnético dentro de uma bobina em que circula corrente elétrica $I$, será gerado no interior desta bobina um campo magnético $H$, sendo que o material 
irá sofrer uma indução magnética $B$. A Figura 3 mostra a curva de histerese deste material.

Figura 3 - Curva de Histerese B x H

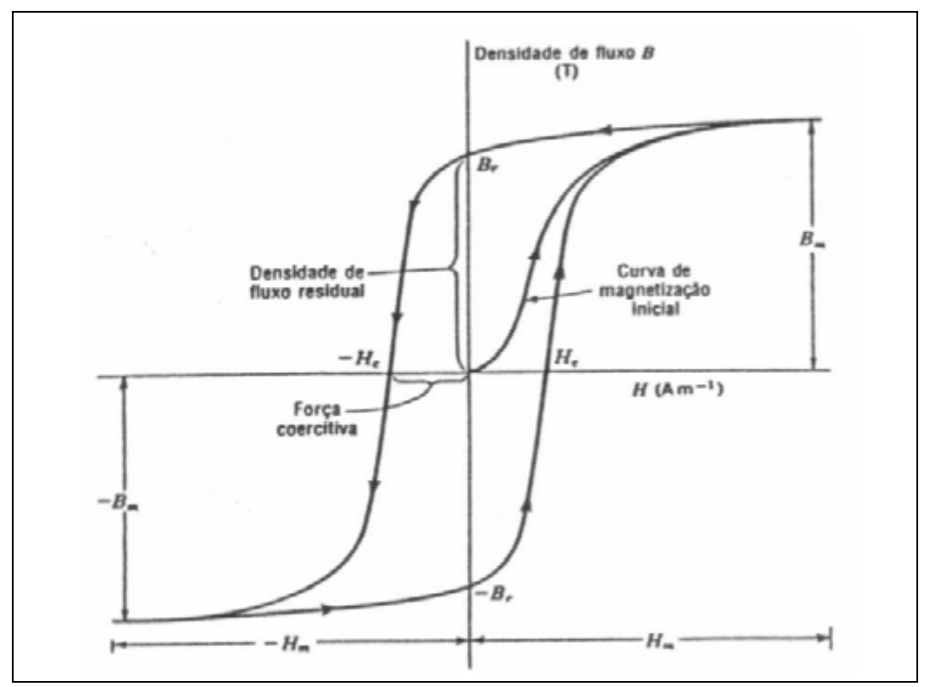

Fonte: Adaptado de CERVA (2014).

O ponto interceptado pela curva de histerese no eixo de $B$, do segundo quadrante, é conhecido como magnetismo remanescente. Tal ponto expressa a indução magnética residual que se mantém no material sem campo magnético aplicado. Nesse ponto, $H$ é igual a zero. $B_{r}$ representa o magnetismo máximo que também é conhecido por retentividade. O ponto interceptado no eixo $H$ do referido quadrante é intitulado de força coercitiva, representando o campo magnético necessário para desmagnetizar o material. Neste ponto $B$ é igual a zero (CERVA, 2014).

\subsubsection{Medições Magnéticas com Anel de Rowland}

Segundo Katz (2005) e Ludke (2009) o Anel de Rowland consiste em um anel toroidal revestido por uma bobina magnetizadora e uma bobina sensora localizada em um dos polos do anel que serve para medir o fluxo magnético gerado no interior do anel. A corrente que passa pelas espiras do enrolamento do primário é proporcional ao campo magnético B do material. Medindo a tensão da bobina e plotando os dados em função da corrente, determinase a curva de histerese para qualquer material ferromagnético. A Figura 4-a apresenta o anel de Rowland construído com material sinterizado. A Figura 4-b apresenta o circuito com anel de Rowland para obtenção das curvas de histerese. 
Figura 4 - (a) Anel de Rowland, (b) Circuito com Anel de Rowland

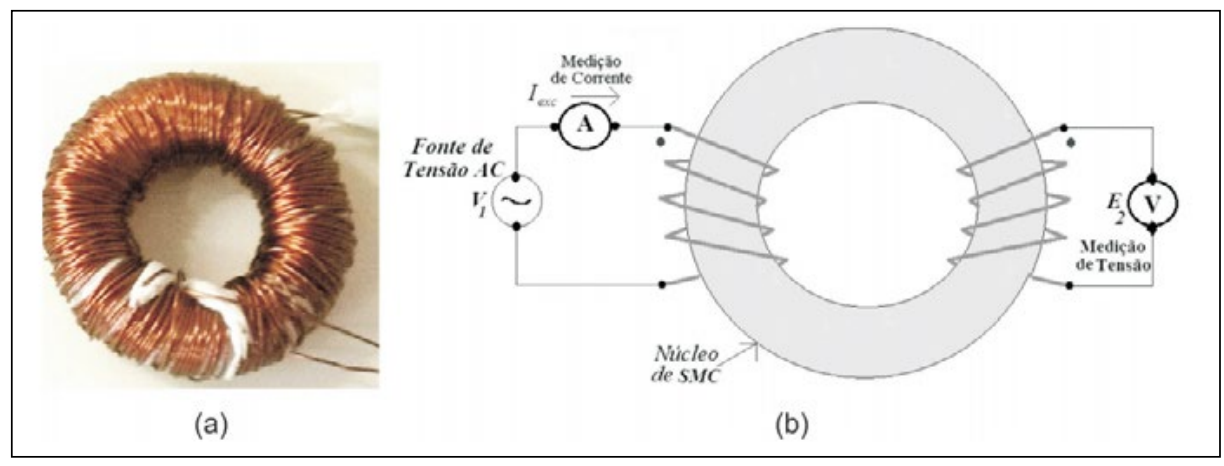

Fonte: CAMPOS (2014).

Utiliza-se um multímetro para medir a força magnetomotriz no enrolamento do secundário e um amperímetro para medir a corrente de excitação do primário. Conhecendo a frequência que é aplicada na bobina, o número de voltas do enrolamento do secundário e a área da seção do núcleo determina-se a máxima densidade de fluxo magnético B (CAMPOS, 2014), conforme apresenta a Equação 1.

$$
B=\frac{4 R_{2} C}{N_{2} \pi w^{2}} V C
$$

Onde:

B Densidade fluxo magnético [T]

C Capacitor do integrador do secundário [F]

$\mathrm{N}_{2} \quad$ Número de espiras do secundário

Vc Tensão induzida no secundário [V]

$\mathrm{R}_{2} \quad$ Resistor do integrador do secundário [ []

w Diâmetro da circunferência do anel [m]

Para determinar a máxima intensidade de campo magnético $H$ (CAMPOS, 2014), utiliza-se a Equação 2.

$$
H=\frac{N_{1} I}{2 \pi R}
$$

Onde:

I Corrente elétrica [A]

$\mathrm{H}$ Intensidade campo magnético máxima $[\mathrm{A} / \mathrm{m}]$

$\mathrm{N}_{1} \quad$ Número de espiras do primário

$\mathrm{R} \quad$ Raio maior [m] 


\subsection{Materiais Moles e Duros}

Determina-se as propriedades magnéticas de um material a partir da sua estrutura eletrônica, cristalina e microestrutura. Os materiais magnéticos são classificados em moles ou duros. Material mole é aquele que é fácil de ser magnetizado ou desmagnetizado. Já o material duro permanece magnetizado ou é um magneto permanente. A Figura 5 apresenta curvas de magnetização e ciclos de histerese característicos desses tipos de materiais (PADILHA, 2000).

Figura 5 - Curvas de magnetização e ciclos de histerese dos materiais (a) moles e (b) duros

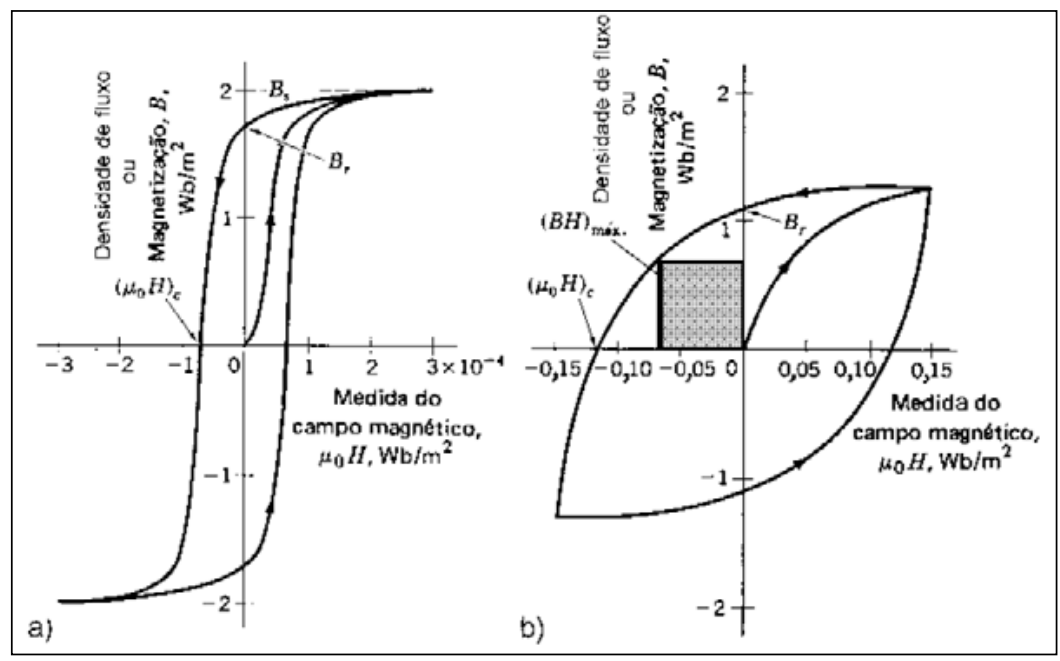

Fonte: PADILHA (2000).

A Figura 5-a. apresenta a curva de magnetização e histerese para materiais magnéticos moles, enquanto que a Figura 5-b. apresenta a curva de magnetização e histerese para materiais magnéticos duros. $\mathrm{O}$ requisito para que um material seja considerado mole é que uma alta magnetização de saturação $B_{s}$ seja produzida por um campo aplicado. O campo necessário para provocar a desmagnetização deverá ser pequeno. Dessa forma a área do ciclo de histerese e a perda de energia por ciclo são pequenas. Materiais magnéticos moles sempre operam na presença de um campo magnético.

Este tipo de comportamento é benéfico para aplicações que envolvam mudanças contínuas na direção de magnetização. Desta forma, utiliza-se materiais magnéticos moles para produção de transformadores, pois estes equipamentos têm eficiência maior se a magnetização não permanece depois que o campo zera. Para esse tipo de aplicação se indica utilizar materiais magnéticos moles de baixa remanência, como ferro puro e aço silício (PADILHA, 2000). 
Materiais magnéticos duros são utilizados em imãs permanentes e devem ter resistência a desmagnetização. Analisando o comportamento da curva de histerese da Figura 5-b conclui-se que esse material tem uma elevada remanência, coercitividade e densidade de fluxo de saturação, baixa permeabilidade inicial e altas perdas de energia por histerese. Possuindo histerese larga, o material apresenta dificuldade em movimentar as paredes de domínio, impedindo a movimentação destas paredes o material eleva sua coercitividade e diminui sua susceptibilidade, assim necessitando de altos valores de campo para desmagnetizar o material (CALLISTER, 2007).

\subsection{Materiais SMC}

Os materiais compósitos magnéticos macios (SMC) são materiais com propriedades eletromagnéticas e que possuem dois ou mais elementos em sua composição. Sua fabricação é via metalurgia do pó (BAGGIO FILHO, 2008). Estes materiais são gerados de minúsculas partículas de pó de ferro cobertas por um material dielétrico, que produz isolação elétrica aos grãos conforme apresenta a Figura 6. O pó de ferro é misturado com material dielétrico e lubrificante, como fósforo por exemplo.

Figura 6 - Representação esquemática do SMC

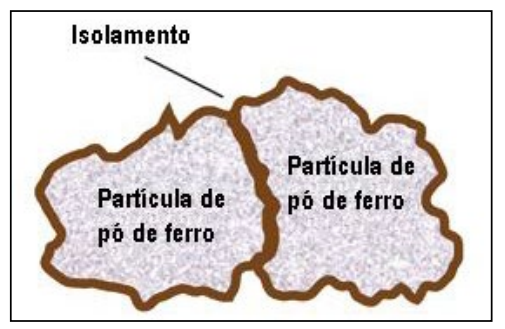

Fonte: HULTMAN (2004).

Esta mistura é colocada em uma matriz e então compactada para gerar peças de material ferromagnético. O método utilizado para compactação é a prensagem axial numa matriz de aço. Após este processo, o material é levado a um forno para fazer com que os grãos de ferro se misturem ao material dielétrico. Recomenda-se a utilização destes materiais para construção de núcleos de equipamentos eletromagnéticos sujeitos a campos magnéticos variáveis, por estes materiais possuírem isotropia magnética e alta resistividade elétrica (CAMPOS, 2014; HOGANAS, 2017).

As características dos compósitos magnéticos macios apresentam diferenças mecânicas e eletromagnéticas dos materiais ferromagnéticos convencionais. O fluxo magnético é o mesmo em todas as dimensões do material. Comparando-se o SMC com o aço laminado verifica-se que a permeabilidade relativa no SMC é bem inferior ao aço laminado. Isto acontece porque o fluxo 
magnético tem que passar pelo material isolante que não é ferromagnético. Já as perdas por ciclo de histerese são maiores no SMC, enquanto que as perdas por correntes parasitas são maiores no aço laminado. Pelas perdas por correntes parasitas no SMC serem menores este material trabalha melhor em altas frequências (RONCHI, 2015).

\subsection{Software LabVIEW}

O LabVIEW é um software que trabalha com linguagem de programação gráfica, conhecida como linguagem G. Ele é recomendado para criação de aplicações flexíveis e sofisticadas de instrumentos que atendam um determinado projeto de pesquisa. Ele permite que se desenvolva aplicações alimentadas com sinais reais, analisando e visualizando dados, criando e prototipando algoritmos, integrando diferentes bibliotecas e linguagens de programação textuais, implementando a aplicação final através de tecnologia disponível no mercado. Tudo isso de forma rápida e com baixo custo de desenvolvimento. A Figura 7-a apresenta a tela de programação G, baseada em blocos de programação. A Figura 7-b apresenta o resultado desta programação que é apresentada ao usuário que irá executar o projeto (NATIONAL INSTRUMENTS, 2012).

Figura 7 - (a) Tela de programação, (b) Resultado da programação

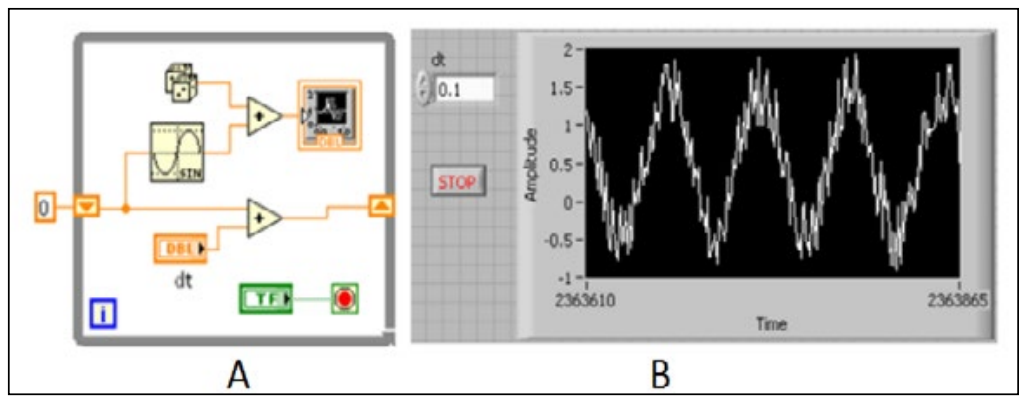

Fonte: NATIONAL INSTRUMENTS (2012).

O LabVIEW possui uma extensa biblioteca capaz de processar sinais e analisar dados. As principais funcionalidades do programa são ajustes de curvas, interpolação e extrapolação, otimização, álgebra linear, probabilidade e estatística, solucionar equações diferenciais, processamento de sinais analógicos e digitais, integração e diferenciação (NATIONAL INSTRUMENTS, 2012).

Segundo National Instruments (2007), o LabVIEW é ideal para construir instrumentos virtuais porque fornece um ambiente de desenvolvimento de projetos de fácil utilização, permitindo que o projetista consiga facilmente operar o programa de instrumentação, controlar o hardware selecionado, analisar os dados adquiridos e exibir os resultados desejados. A ferramenta 
permite que o projetista personalize cada painel com botões, knobs, seletores, gráficos, deixando a aplicação fácil de ser visualizada e interativa.

\subsection{Software FEMM}

O principal objetivo da metodologia de elementos finitos é solucionar equações diferenciais para diversos tipos de entradas. Ao dividir o problema em muitas regiões em formato de triângulos com áreas conhecidas, acaba-se deixando o problema com resolução mais simples. O problema de álgebra linear com milhares de variáveis é formado a partir do processo de discretização. O software FEMM 4.2 (Finite Element Method Magnetics) é composto por um conjunto de programas capaz de resolver problemas de baixa frequência eletromagnética com planos bidimensionais com simetria axial. A Figura 8 apresenta as variáveis de entrada e saída do aplicativo (SCHAEFFER, 2008; CERVA, 2014; DIAS, 2014).

Figura 8 - Variáveis de entrada e saída do FEMM

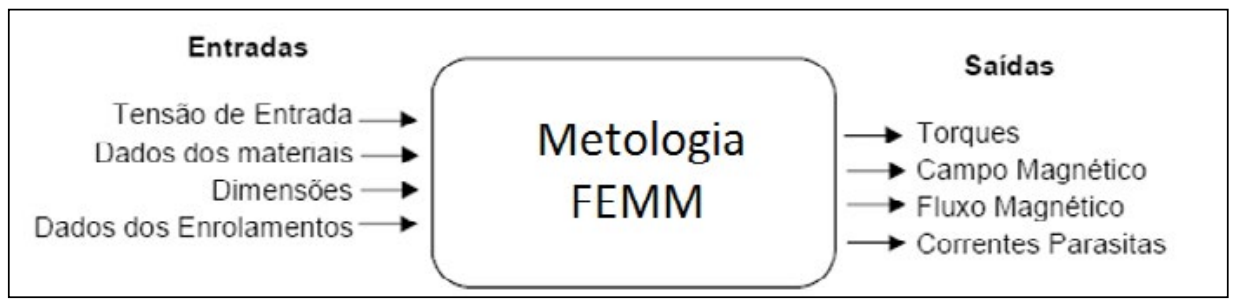

Fonte: Adaptado de SCHAEFFER (2008).

O programa é divido em Interative Shell, Triangle.exe, Solvers. A primeira é uma interface múltipla com pré e pós processamento para diversos tipos de problemas. Essa parte contém uma interface CAD (Computer Aided Designed) responsável por definir as propriedades do material e as condições de limite. A segunda é responsável por dividir a região a ser solucionada em um grande número de triângulos com áreas conhecidas, possibilitando a resolução do problema por elementos finitos. A última é um algoritmo capaz de resolver problemas magnéticos e eletroestáticos. Cada parte utiliza os dados préconfigurados resolvendo as equações diferenciais de Maxwell obtendo os valores desejados (SCHAEFFER, 2008; CERVA, 2014; DIAS, 2014).

\subsection{Software PSIM}

De acordo com PSIM (2006) e Gomes (2012), o PSIM é um software de simulação capaz de analisar uma forma de onda semelhante a apresentada em uma tela de osciloscópio. Este software é muito utilizado para analisar malhas de controle, amplificadores analógicos e digitais, curvas de histerese, entre tantos outros. Este simulador pode ser utilizado em praticamente todos os circuitos 
analógicos e digitais existentes. Para realizar uma simulação deve-se seguir os seguintes passos:

- Criação de esquemático de um circuito a ser simulado;

- Realização da simulação;

- Análise dos resultados.

A Figura 9-a apresenta um oscilador de onda quadrada simulado neste software. Enquanto que a Figura 9-b apresenta o resultado da simulação.

Figura 9 - (a) Oscilador de onda quadrada e (b) Resultado da simulação do oscilador de onda quadrada

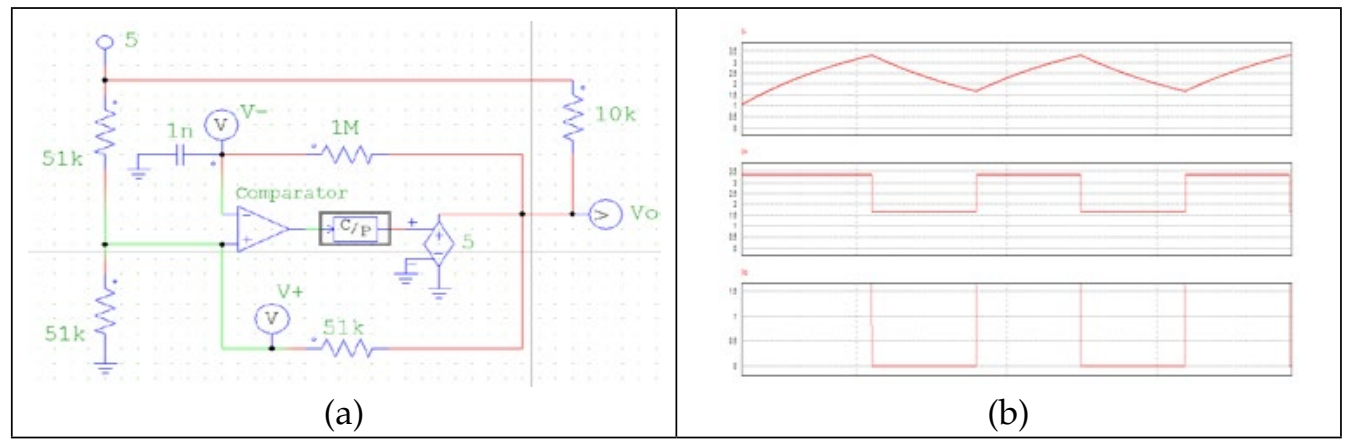

Fonte: Autor (2017).

O circuito da Figura 9-a é alimentado com um sinal de tensão contínua de $5 \mathrm{~V}$ de amplitude. Medindo-se a entrada inversora do amplificador operacional comparador, analisa-se uma onda quadrada, com offset de $2,5 \mathrm{~V}$ e $1 \mathrm{~V}$ de amplitude. Enquanto que na entrada não inversora é medido um sinal de onda triangular com offset $1 \mathrm{~V}$ e $2 \mathrm{~V}$ de amplitude. Na saída do circuito, encontra-se uma onda quadrada de 1,5 V de amplitude. Com este exemplo, comprova-se que o PSIM é um excelente software de simulação de circuitos eletrônicos, no qual o usuário pode personalizar o circuito conforme sua necessidade, e assim, avaliar cada aspecto do sistema através de gráficos e valores numéricos.

\section{METODOLOGIA}

O presente trabalho tem como objetivo realizar um estudo do comportamento eletromagnético de diferentes tipos de materiais macios sinterizados, que são utilizados em núcleos de máquinas elétricas, como motores e transformadores. Dentre os aspectos mais importantes que são avaliados para cada material, estão as curvas de histerese, densidade de fluxo magnético e intensidade do campo magnético. Para tanto, desenvolveu-se um hardware e um aplicativo de instrumentação virtual dentro do software LabVIEW. A Figura 10 apresenta o fluxograma do processo de análise do material. 
Figura 10 - Fluxograma do processo de análise do material

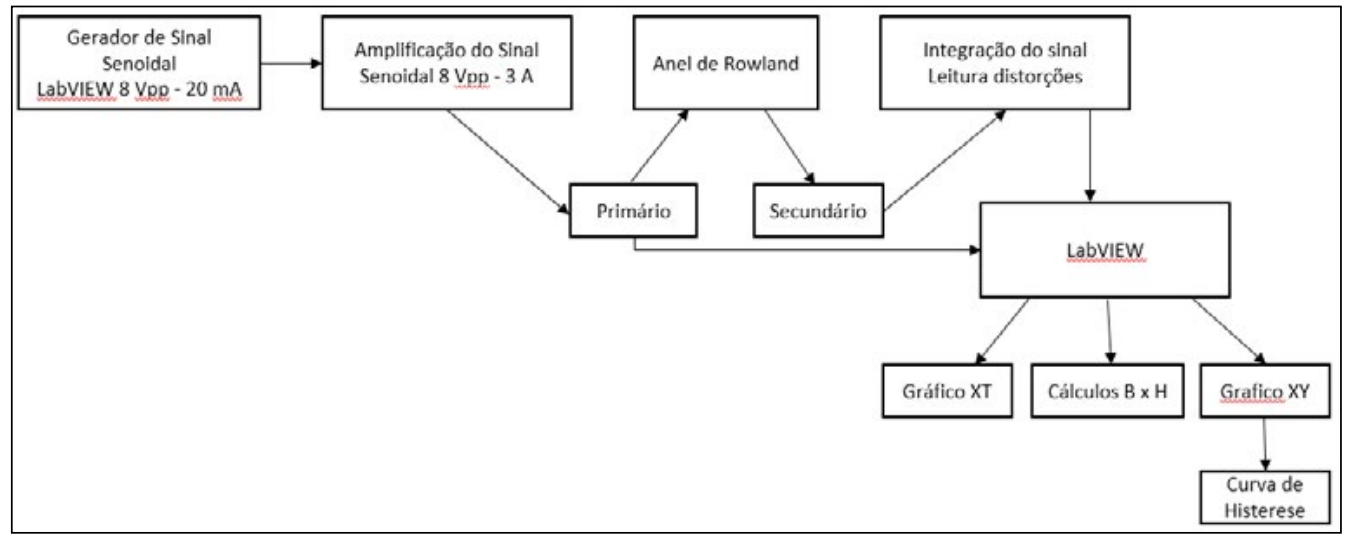

Fonte: Autor.

O aplicativo de instrumentação virtual desenvolvido no LabVIEW, gera um sinal senoidal de $8 \mathrm{Vpp}$ e $20 \mathrm{~mA}$. Este sinal é enviado a um circuito amplificador que entrega $8 \mathrm{Vpp}$ e $3 \mathrm{~A}$ na saída. O sinal amplificado é então aplicado ao primário do anel de Rowland a ser testado. Este mesmo sinal do primário é lido no canal 1, do bloco de entradas e saídas do LabVIEW, que envia o sinal para o aplicativo desenvolvido neste software. Na saída do secundário do anel é coletado o sinal e enviado a um outro circuito integrador, responsável por ler as distorções e enviar ao canal 2 do bloco de entradas e saídas, no qual será também enviado ao aplicativo do software. Este aplicativo recebe as informações do canal 1 e 2, bem como as informações do anel, que o usuário que realizará os testes deverá inserir manualmente, como número de espiras do primário e secundário, resistor utilizado no primário do anel, raio e diâmetro da circunferência do anel. Com estas informações o aplicativo mostra na tela do computador a curva de histerese e os valores da intensidade e densidade de campo magnético.

\subsection{Projeto do circuito eletrônico}

Desenvolveu-se um circuito eletrônico que lê um sinal senoidal de $8 \mathrm{~V}$ e $20 \mathrm{~mA}$, gerado pelo LabVIEW, e amplifica na saída o mesmo sinal em $8 \mathrm{~V}$ e $3 \mathrm{~A}$. O hardware conta com três amplificadores operacionais, sendo um diferenciador, um inversor e um integrador para garantir os $8 \mathrm{~V}$ na saída, responsáveis por manter a realimentação do circuito.

Para amplificação da corrente, utilizou-se três estágios de push pull, com dois transistores em cada estágio, um NPN e outro PNP. Os transistores escolhidos para os estágios 1,2 e 3 respectivamente, foram BC338 e BC339, TIP41 e TIP42, N23055 e MJ2955. Estes transistores foram escolhidos em virtude de suas correntes de base e de coletor casarem com a corrente de base e coletor do 
estágio seguinte. O projeto foi dimensionado para amplificar correntes até 15 A e produzir $115 \mathrm{~W}$ de potência, porém neste projeto está limitado à corrente máxima das fontes de alimentação (3 $\mathrm{A}$, com tensão de $+/-24 \mathrm{~V})$.

Para leitura do sinal de tensão do secundário do anel, utilizou-se um amplificador operacional com a configuração integradora. Com um resistor de $10 \mathrm{k} \Omega$ e um capacitor de $100 \mathrm{nF}$ interligados neste amplificador, garantiu-se a leitura esperada para este sinal. Após o projeto do circuito, foi utilizado a técnica de fabricação de circuito impresso, para reproduzir o circuito em placa de fibra de vidro. A Figura 11 apresenta o esquemático completo deste circuito.

Figura 11 - Esquemático do circuito eletrônico

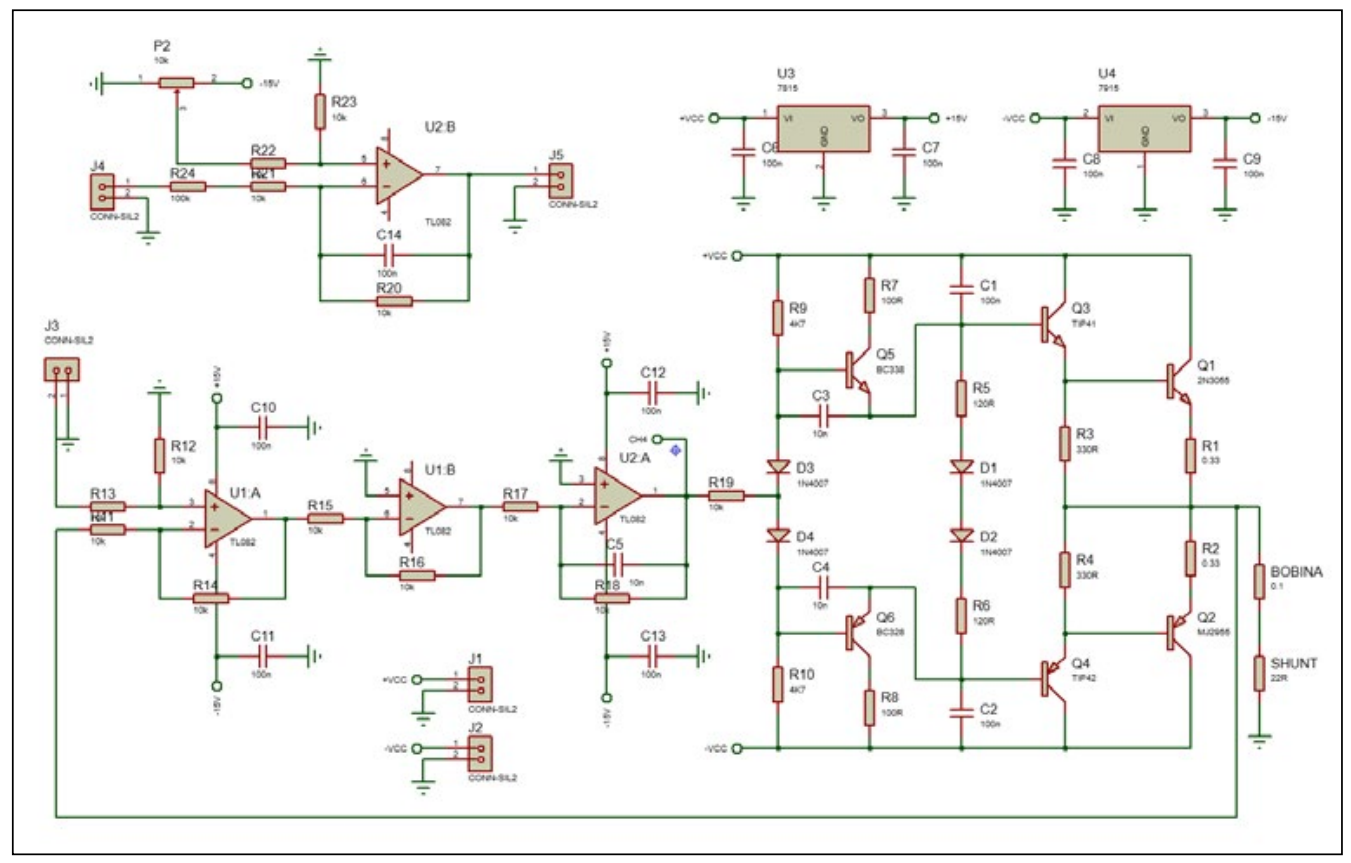

Fonte: Autor.

Após montagem do circuito, o mesmo foi colocado dentro de um gabinete de madeira, juntamente com suas duas fontes de alimentação. Neste gabinete, colocou-se 10 bornes para ligação dos canais 1 e 2, inicialmente ligados em osciloscópio Teketronix, modelo TDS 1002B, e posteriormente no bloco de entradas e saídas do LabVIEW; o resistor de potência em série com o primário para limitação de corrente; o gerador de sinais senoidais, inicialmente da Agilent, modelo 33220A, e posteriormente do gerador do LabVIEW; os fios terra, primário e secundário do anel de Rowland. A Figura 12-a ilustra o equipamento montado e a Figura 12-b apresenta a placa de circuito impresso do push-pull. 
Figura 12 - (a) Projeto montado em gabinete de madeira e (b) Placa de circuito impresso do Push-Pull

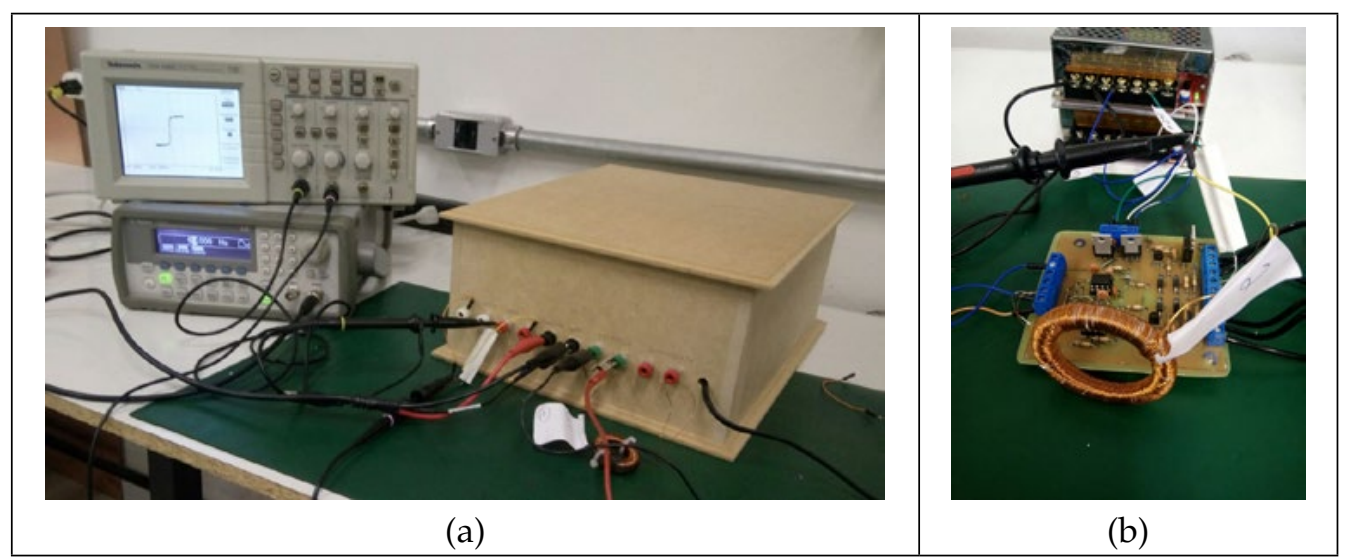

Fonte: Autor.

\subsection{Funcionamento do aplicativo}

Desenvolveu-se um aplicativo de instrumentação virtual no software LabVIEW. A frequência de amostragem foi de $25 \mathrm{kHz}$. Este aplicativo recebe os sinais analógicos de tensão do primário e secundário do anel a ser testado, lido a partir dos canais 1 e 2 do bloco de entradas e saídas do LabVIEW. O usuário insere no aplicativo o número de espiras do primário e secundário do anel, o raio e o diâmetro da circunferência do núcleo, bem como o valor do resistor ligado em série com a bobina do primário para limitação de corrente. Todos as unidades dos valores inseridos devem estar no Sistema Internacional de Medidas. Com estes dados, o aplicativo calcula os valores de intensidade de campo e densidade de campo magnético. Além disso, plota na tela do computador o gráfico da curva de histerese do material e a curva dos sinais do primário e secundário. A Figura 13 apresenta a tela do aplicativo. 
Figura 13 - Aplicativo Histeresímetro

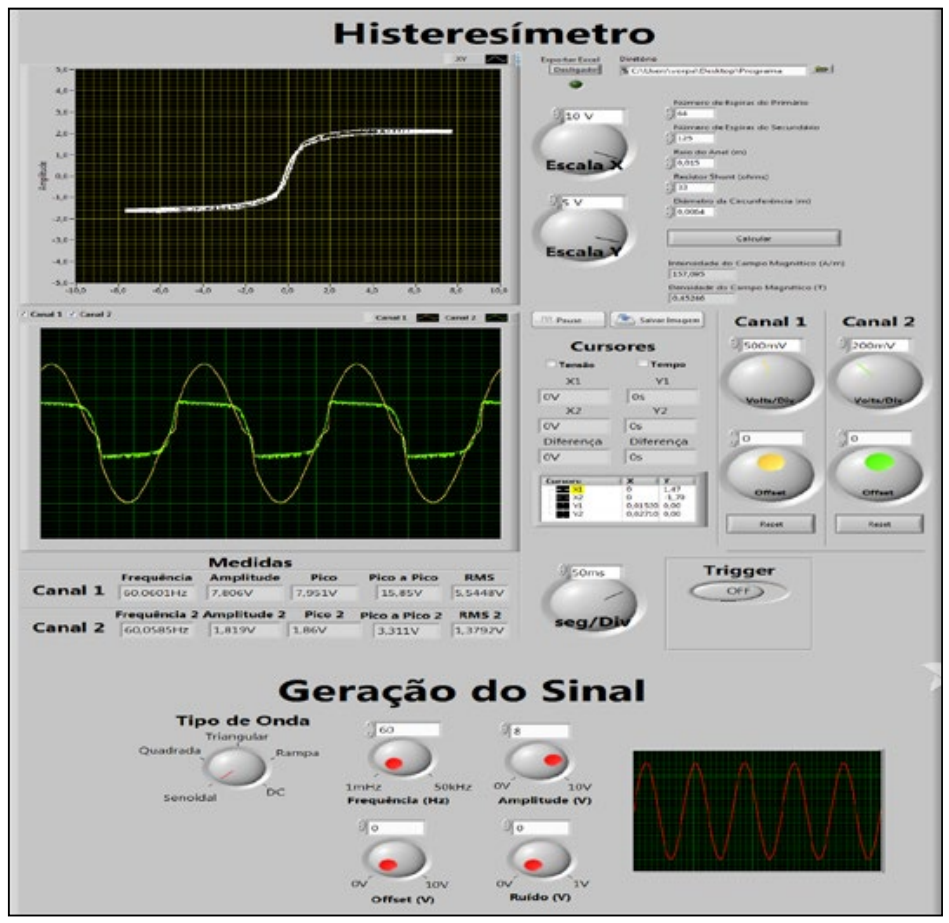

Fonte: Autor.

Tanto no gráfico da curva de histerese, quanto no gráfico das curvas do primário e secundário do anel inseriu-se as funções de ajustar as escalas $X$ e Y. No gráfico das curvas do primário e secundário ainda é possível dar offset no sinal, ajustar quantos segundos por divisão o usuário deseja visualizar, acionar a função Trigger, posicionar cursores de tensão e de tempo e visualizar as medidas de frequência, amplitude, pico, pico a pico e tensão RMS.

Outra funcionalidade implementada é a opção de exportar a curva de histerese gerada na tela do computador para o software de planilhas, como por exemplo o Excel. O usuário ativa na tela do software a opção "Exportar Excel" e a partir do momento que a luz indicadora da função estiver ligada, todos os dados referentes a curva de histerese serão enviados a um arquivo externo em formato ".lvm" que pode ser aberto em qualquer aplicativo de planilha eletrônica.

Além destas funções, inseriu-se um gerador de sinais de $1 \mathrm{mHz}$ a 50 $\mathrm{kHz}$ de frequência e 0 a $10 \mathrm{~V}$ de tensão. Este gerador é capaz de gerar onda senoidal, quadrada, triangular, rampa e contínua. Ele ainda conta com a função offset e é capaz de gerar um ruído de 0 a $1 \mathrm{~V}$. Este gerador ainda exibe na tela do aplicativo a forma de onda que está sendo gerada. 


\subsection{Validação da proposta}

A proposta foi validada através da comparação dos resultados dos materiais analisados via LabVIEW, FEMM e PSIM, como curvas de histerese, valores de campo magnético e densidade de fluxo magnético, com um traçador de curvas comercial da marca GLOBALMAG, modelo TLMP-FLX-D, conforme apresenta a Figura 14.

Figura 14 - (a) Equipamento TLMP-FLX-D e (b) Curva de histerese gerada pelo software

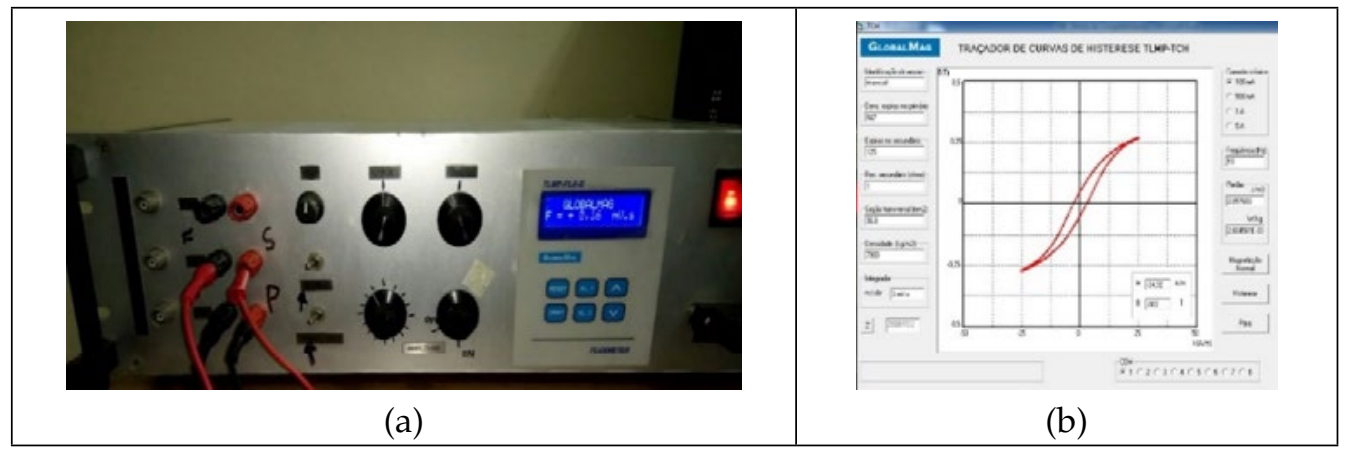

Fonte: Autor.

As curvas obtidas com o aplicativo de instrumentação virtual, bem como as obtidas com o equipamento da GLOBALMAB, foram exportadas para uma planilha eletrônica de Excel e sobrepostas. Desta maneira verificou-se o erro padrão do equipamento desenvolvido neste trabalho. Os resultados obtidos nos softwares FEMM e PSIM serviram de modelo para conclusão da análise dos materiais testados.

\section{RESULTADOS E CONCLUSÕES}

Neste tópico são apresentados os resultados e conclusões obtidas nos testes realizados em um material magnético macio. Neste material são apresentadas as simulações realizadas nos softwares FEMM 4.2 e PSIM 9.0, bem como os as tabelas com os resultados das curvas de histerese no aplicativo desenvolvido em LabVIEW e no equipamento de validação do sistema da fabricante GLOBALMAG.

\subsection{Material 1}

A composição do material 1 é ferrite. Abaixo são apresentadas as especificações técnicas do anel de Rowland utilizando este material, bem como a corrente e a frequência do sinal senoidal aplicado no primário, a tensão lida no secundário, o capacitor e o resistor do circuito integrador. 
- Composição do material: Ferro puro

- Número espiras primário: 64 espiras

- Número de espiras do secundário: 125 espiras

- Raio do anel: 0,015 m

- Diâmetro do núcleo: 0,0064 m

- Área da seç̧ão do núcleo: 0,0368 m

- Corrente aplicada no primário: 0,232 A

- Frequência do sinal senoidal: $60 \mathrm{~Hz}$

- Tensão lida no secundário: $1,86 \mathrm{~V}$

- Capacitor integrador: $100 \mathrm{nF}$

- Resistor integrador: $10 \mathrm{k} \Omega$

A partir destas informações calcula-se a intensidade de campo magnético $\mathrm{H}$ máxima, medido em $\mathrm{A} / \mathrm{m}$ e a densidade de fluxo magnético máxima, medido em T, utilizando-se, respectivamente, as Equações 1 e 2, representadas no tópico 2 deste trabalho.

$$
\begin{aligned}
& B=0,45 \mathrm{~T} \\
& H=157,54 \mathrm{~A} / \mathrm{m}
\end{aligned}
$$

Desta forma, utilizando-se o aplicativo desenvolvido em LabVIEW obteve-se a curva de histerese do material. A Figura 15 apresenta a tela do aplicativo. 
Figura 15 - Tela do aplicativo Histeresímetro para material 1

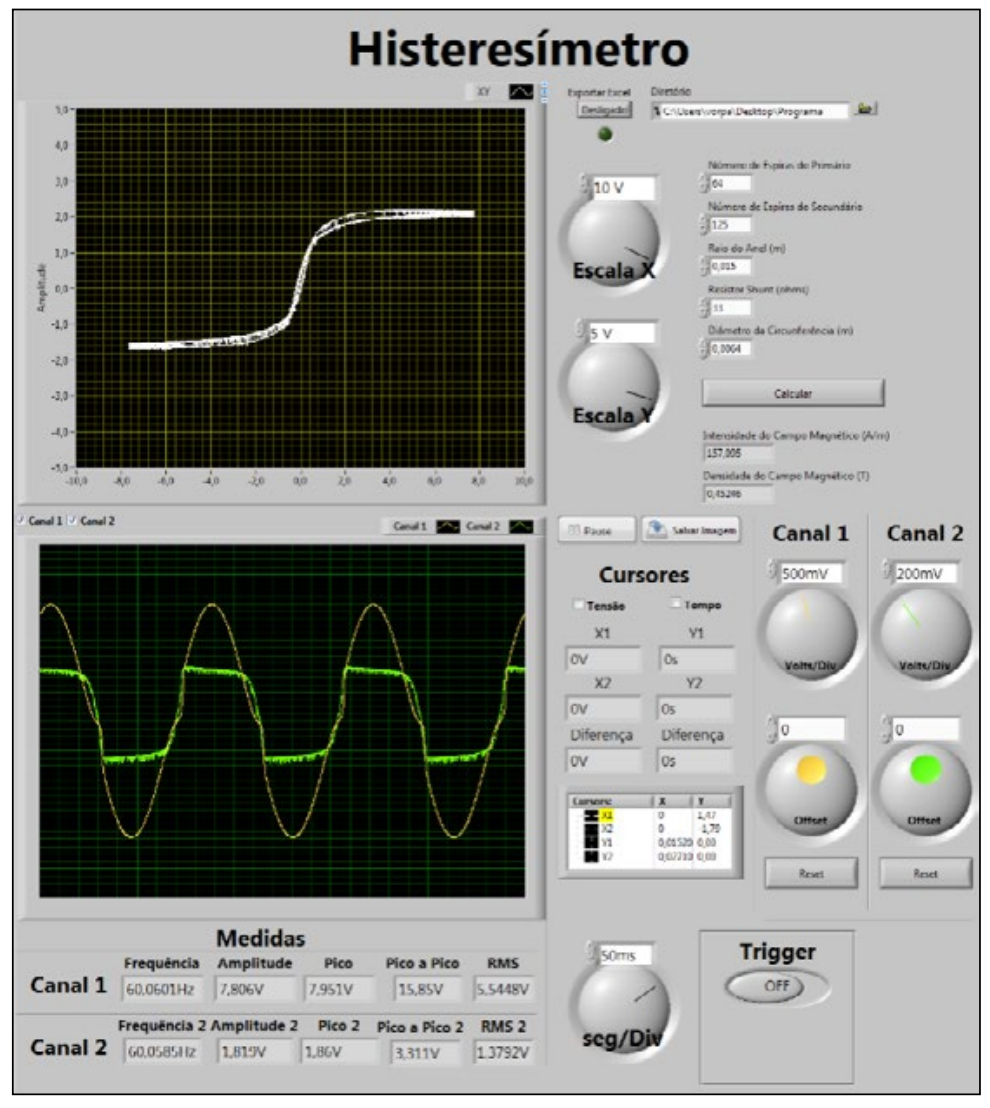

Fonte: Autor.

Nesta tela, observa-se a curva para um material mole, que satura com baixas correntes e possui a área do ciclo de histerese reduzida, da mesma forma que a perda de energia por ciclo, característica típica deste tipo de material. $\mathrm{O}$ aplicativo também apresenta os valores de $H$ e $B$ que condizem com os valores calculados anteriormente. Os valores de intensidade de campo magnético e densidade de fluxo magnético encontrados são coerentes com o material utilizado. Para validação desta curva, exportou-se a mesma para uma planilha eletrônica, onde inseriu-se a curva obtida com o equipamento traçador de curva de histerese da GLOBALMAG, fazendo-se uma sobreposição das duas curvas, conforme ilustra a Figura 16. 
Figura 16 - Curva comparativa do material 1

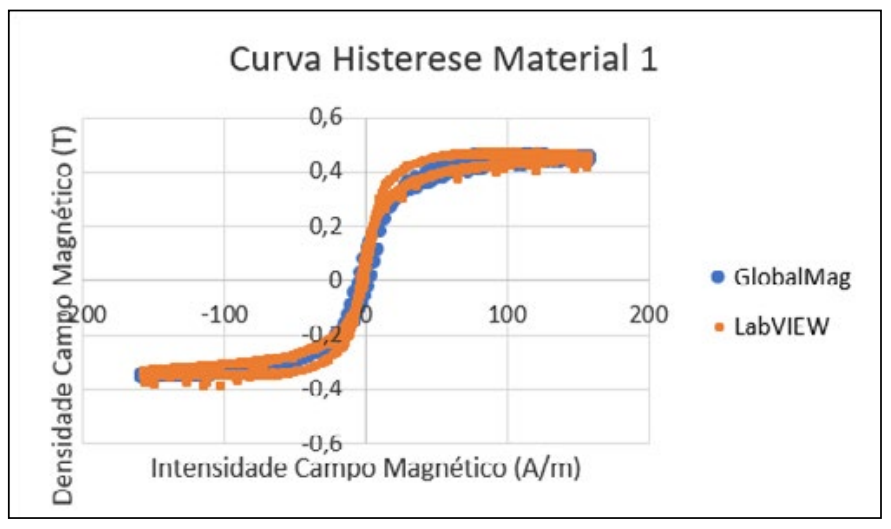

Fonte: Autor.

Mensurou-se os valores de densidade de fluxo magnético remanescente $\left(B_{r}\right)$, densidade de fluxo magnético máximo e intensidade de campo magnético máximo, tanto da curva obtida pelo equipamento GLOBALMAG quanto do aplicativo do LabVIEW, afim de comparar os principais pontos das duas curvas, verificando sua veracidade, conforme apresenta a Tabela 1 . A coercitividade do material (Hc) não foi possível mensurar por limitações do hardware, uma vez que no circuito do integrador do secundário utilizou-se capacitor e resistor de valores fixos e não variáveis, desta forma, não permitindo a leitura correta da coercitividade. Caso pudesse variar esta relação utilizando-se um capacitor e um resistor variável, poder-se-ia calibrar o equipamento ajustando-se o valor da coercitividade. Esta limitação fica acentuada em função da corrente necessária para saturar o material ser muito baixa.

Tabela 1 - Comparativo de B x H do material 1

\begin{tabular}{l|l|l|l|l}
\hline \multicolumn{2}{l|}{ GLOBALMAG } & \multicolumn{2}{l|}{ LABVIEW } & Erro $(\%)$ \\
\hline Br & 0,119 & Br & 0,111 & $6,723 \%$ \\
\hline Bmáx & 0,463 & Bmáx & 0,468 & $1,079 \%$ \\
\hline Hmáx & 159,013 & Hmáx & 157,864 & $0,722 \%$ \\
\hline
\end{tabular}

Fonte: Autor.

Os valores calculados estão de acordo com os encontrados pelo aplicativo desenvolvido no LabVIEW e são validados pelos resultados obtidos a partir traçador de curvas de histerese da GLOBALMAG, conforme apresentado na Tabela 1.

Utilizando-se o software FEMM 4.2, desenhou-se o anel de Rowland utilizando-se das mesmas especificações técnicas do material e os mesmos 
valores de corrente e frequência aplicadas no primário do anel. As Figuras 17-a e 17-b ilustram, respectivamente, o comportamento do anel para intensidade de campo magnético $\mathrm{H}$ e densidade de fluxo magnético $\mathrm{B}$.

Figura 17 - (a) Comportamento do material 1 para $\mathrm{H}$ e (b) comportamento do material 1 para B

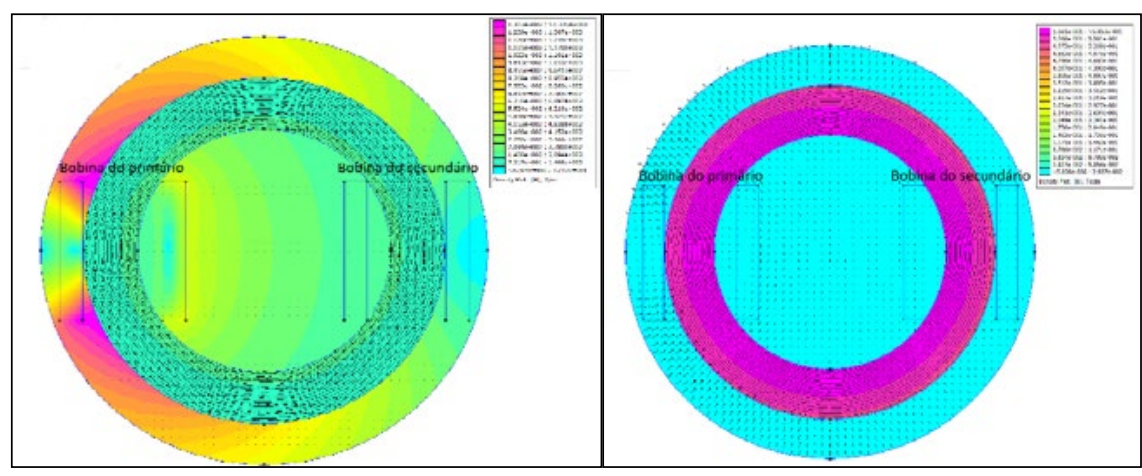

Fonte: Autor.

Abaixo apresenta-se os valores numéricos encontrados pela simulação.

Corrente Total: $0,23 \mathrm{~A}$

Fluxo magnético: 0,023 Wb

Densidade de fluxo magnético: 0,468 T

Intensidade do campo magnético: 158,32 A/m
Potência ativa: 0,151 W

Potência reativa: $0,918 \mathrm{VAr}$

Potência Aparente: 0,930 VA

Tensão induzida no secundário: $1,86 \mathrm{~V}$

A partir desta simulação verifica-se que a intensidade de campo magnético é bem mais intensa no lado do primário, onde é aplicada a corrente elétrica. As linhas de campo magnético se movimentam no sentido horário do núcleo, percorrendo uniformemente o material. Já densidade de fluxo magnético apresenta maior intensidade na superfície do núcleo, perdendo fluxo magnético à medida que se aproxima do interior do anel. As linhas de fluxo magnético formam um campo girante no primário do anel, induzindo fluxo ao secundário. Também se verifica que os valores numéricos de $B$ e $H$ condizem com os encontrados no aplicativo desenvolvido no LabVIEW, desta forma servindo também de validação a este trabalho.

Da mesma forma que no FEMM 4.2, utilizando-se o software PSIM 9.0, desenhou-se o esquemático do circuito eletrônico do anel de Rowland, com todas as especificações utilizadas até o momento para determinar a curva de histerese do material. Realizando-se comparações com as curvas obtidas no aplicativo desenvolvido em LabVIEW e no equipamento padrão da GLOBALBAL. A Figura 18-a apresenta o esquemático do circuito desenhado no PSIM e a curva de histerese do material simulado é ilustrada pela Figura 18-b. 
Figura 18 - (a) Esquemático do circuito no PSIM e (b) Curva de histerese material 1

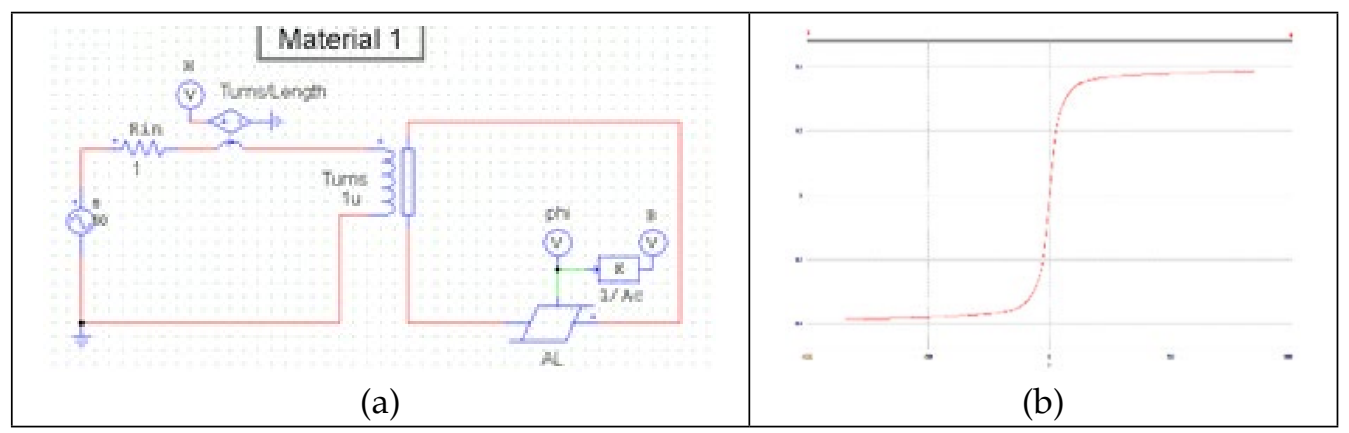

Fonte: Autor.

Desta forma, conclui-se que o material 1, a $60 \mathrm{~Hz}$, satura com baixas correntes e possui a área do ciclo de histerese e a perda de energia por ciclo pequenas, resultados já esperados para este tipo de liga metálica. Além disso, utilizou-se os softwares FEMM 4.2 e PSIM 9.0 para determinar o comportamento eletromagnético do anel e as curvas de histerese. Aplicando estas simulações ao anel 1, conclui-se que a intensidade de campo magnético é bem mais intensa no lado do primário, onde é aplicada a corrente elétrica. As linhas de campo magnético se movimentam no sentido horário do núcleo, percorrendo uniformemente o material. Já a densidade de fluxo magnético apresenta maior intensidade na superfície do núcleo, perdendo fluxo magnético à medida que se aproxima do interior do anel. As linhas de fluxo magnético formam um campo girante no primário do anel, induzindo fluxo ao secundário. Além disso, as curvas de histerese e os valores de densidade de fluxo magnético e intensidade de campo magnético são análogos aos encontrados pelo aplicativo desenvolvido no LabVIEW. Utilizando um equipamento de medição padrão da GLOBALMAG, modelo TLMP-FLX-D, validou-se todas as curvas geradas pelo aplicativo desenvolvido neste trabalho.

Em trabalhos futuros, a fim de calibrar o equipamento ajustandose o valor da coercitividade para cada material testado, sugere-se que no hardware desenvolvido para amplificar os sinais aplicados no primário e ler as distorções do secundário dos anéis, seja substituído o capacitor e resistor fixos do integrador, por capacitor e resistor variáveis. Os resultados obtidos com o equipamento atual variam conforme as características eletromagnéticas dos materiais, frequência e corrente aplicada nos mesmos. Fazendo com que alguns tipos de resultados sejam mais exatos que outros, dependendo do material utilizado e do sinal que é aplicado nele. Inserindo-se o capacitor e resistor variável é possível melhorar a exatidão dos resultados, variando a relação RC do integrador. Para compor a exatidão do equipamento de medida, sugere-se futuramente uma análise das incertezas do equipamento proposto, 
tendo como referência o equipamento comercial adotado na metodologia. Além disso, orienta-se avaliar o produto energético máximo, para quantificar a dimensão do volume do material com o material macio, avaliando-se o erro com a maciez do material. Recomenda-se também, inserir uma programação no aplicativo do LabVIEW capaz de traçar a curva de magnetização do material. Esta curva é importante para determinação das características magnéticas dos materiais. Finalmente, sugere-se ainda realizar testes com outras ligas metálicas sinterizadas, de modo a avaliar o comportamento encontrado com as ferramentas propostas neste projeto.

\section{REFERÊNCIAS}

BARBOSA, R. O. Instrumentação virtual com LabVIEW em laboratório experimental de eletrônica. Trabalho de Conclusão de Curso de Engenharia de Controle e Automação. Centro Universitário Univates. Lajeado, Rio Grande do Sul, 2016.

BAGGIO FILHO, N. F. Estudo e desenvolvimento de um atuador planar com enrolamentos planificados e núcleo de material ferromagnético compósito. Dissertação de Mestrado em Engenharia Elétrica. UFRGS. Porto Alegre, Rio Grande do Sul. 2008. Disponível em: <http://hdl.handle.net/10183/13791>. Acesso em: 22 mai. 2017.

BIANCHINI, D. et al. O ensino de engenharia por meio de laboratórios virtuais de eletrônica: uma reflexão entre a montagem no protoboard e a simulação. Anais do XXXIV COBENGE. Passo Fundo, Set. 2006. Disponível em: https:/ / www. researchgate.net/publication/242676686_O_ENSINO_DE_ENGENHARIA_POR_ MEIO_DE_LABORATORIOS_VIRTUAIS_DE_ELETRONICA_UMA_REFLEXAO_ ENTRE_A_MONTAGEM_NO_PROTOARD_E_A_SIMULACAO>. Acesso em: 19 mai. 2017.

CALLISTER, W. D. Materials Science and Engineering. $7^{\mathrm{a}}$ ed. John Wiley \& Sons, Inc. USA, 2007.

CAMPOS, L. et al. Caracterização das propriedades magnéticas de materiais compósitos magnéticos macios. Revista de Iniciação Científica. ULBRA. Canoas, Rio Grande do Sul, 2014. Disponível em: < http://www.periodicos.ulbra.br/index.php/ ic/article/viewFile/1032/969>. Acesso em: 28 mai. 2017.

CERVA, L. L. Desenvolvimento de uma máquina síncrona com imãs permanentes e núcleo sintetizado utilizada em aerogerador. Tese de Doutorado. UFRGS. Porto Alegre, Rio Grande do Sul, 2014.

CULLITY, B. Introduction of Magnetic Materials. USA; Massachusetts, 1967.

DIAS, M. M., SCHAEFFER, L., DIAS, A. M., CÉSAR, J. L. Novas Perspectivas das Máquinas Elétricas Trifásicas a Partir da Utilização de Materiais Magnéticos Macios Sinterizados. Novo Hamburgo: Revista Liberato, v. 6, n. 6, 2005. p. 56-65. 
DIAS, M. M. et al. Aplicação de materiais magnéticos macios sintetizados em máquinas elétricas rotativas síncronas. 2014. Tecnologia e Tendências. FEEVALE. Vol 10, No2, $2^{\circ}$ Semestre, Novo Hamburgo, 2014.

FERREIRA, R. et al. Virtualização de instrumentos industriais utilizando o LabVIEW. Dept. de Eng. Elétrica e Sistemas de Potência, UFPE - Universidade Federal de Pernambuco. VIII Conferência Internacional de Aplicações Industriais Induscon. Ago. 2008.

GOMES, I. S. F. et al. PSIM. Programa de Educação Tutorial. Universidade Federal do Ceará. Fortaleza, Ceará, 2014.

GRIFFITHS, D. J.; COLLEGE, R. Introduction to Electrodynamics. USA; New Jersey, V. 4, 2012.

HILLEBRANDS, B.; OUNADJELA, K. Spin Dynamics in Confined Magnetic Structures. Alemanha; Springer-Verlag Berlin Heidelberg, v. I, 2002.

HOGANAS, A. B. Compostos Magnéticos Macios (Soft Magnetic Composites SMC). Disponível em: <https:/ /www.hoganas.com/> . Acesso em: 28 mai. 2017.

HULTMAN L.O.; JACK A.G. Soft magnetic composites - motor design issues and applications. Proceedings of the International Conference on Powder Metallurgy and Particulate Materials, p. 194-204, Princeton, NJ, USA. 2004.

KATZ, R. Physics, Magnetic Properties of Matter. Chapter 33. University of Nebraska. EUA; Lincoln, 2005.

LUDKE, E. Estudando campos magnéticos e histerese com um anel de Rowland. Departamento de Física. UFSM. Santa Maria, Rio Grande do Sul, 2010.

LUNA, W. H. D. Desenvolvimento de imãs de Nd-Fe-B pelo processo MPI aplicados em máquinas elétricas (motores). Tese de Doutorado. UFRGS. Porto Alegre, Rio Grande do Sul, 2012.

NASAR, S.A. Handbook of Electric Machines. EUA; Nova York, McGraw-Hill, 1987.

NATIONAL INSTRUMENTS. Vantagens da utilização do LabVIEW em pesquisas acadêmicas. Fev. 2012. Disponível em: < http:/ / www.ni.com/white-paper/8534/ pt/>. Acesso em: 12 mai. 2017.

PADILHA, A. F. Materiais de Engenharia Microestrutura e Propriedades. Hemus. Curitiba, Paraná, 2000.

PSIM. Tutorial on How to Define the Saturable Core Element. Powersim Inc. 2006. Disponível em: www.powersimtech.com. Acesso em: 24 set. 2017.

RONCHI, F. P. Construção e análise do desempenho de um motor de corrente continua utilizando materiais magnéticos macios a partir da metalurgia do pó. 
Dissertação de Mestrado em Engenharia de Minas, Metalurgia e de Materiais. UFRGS. Porto Alegre, Rio Grande do Sul, 2015.

SCHAEFFER, L. et al. Estudo comparativo de ligas magnéticas através de simulação por elementos finitos. Universidade Federal do Rio Grande do Sul, Porto Alegre, 2008.

ZAHN, M. et al. Electromagnetic Fields and Energy. MIT Open Course Ware. Massachusetts Institute of Technology. Massachusetts, EUA, 2012. Disponível em: https:/ /ocw.mit.edu/resources/res-6-001-electromagnetic-fields-and-energyspring-2008/. Acesso em: 09 set. 2017. 